\title{
MONOTONIC DECAY OF SOLUTIONS OF PARABOLIC EQUATIONS WITH NONLOCAL BOUNDARY CONDITIONS*
}

BY

\section{AVNER FRIEDMAN ${ }^{1}$}

Purdue University

1. Introduction. In recent papers [3, 4] Day considered parabolic equations in one space dimension with boundary conditions

$$
u(-a, t)=\int_{-a}^{a} f(x) u(x, t) d x, \quad u(a, t)=\int_{-a}^{a} g(x) u(x, t) d x .
$$

The solution $u$ represents the entropy in a quasi-static theory of thermoelasticity; for a derivation of the model see $[1,2]$. Day proved that if

$$
\int_{-a}^{a}|f(x)| d x<1, \quad \int_{-a}^{a}|g(x)| d x<1,
$$

then the maximum modulus of the entropy is decreasing in time, that is,

$$
U(t) \equiv \max _{-a \leqslant x \leqslant a}|u(x, t)|
$$

is decreasing in $t$. An example in [4] shows that if both inequalities in (1.1) are reversed, then, in general, $U(t)$ may increase exponentially with $t$.

The proof of (1.2) is based on estimating integrals of the form $\int_{-a}^{a} P(x) u^{2 m}(x, t) d x$ with suitably chosen $P(x)$.

In this paper we extend the assertion (1.2) to general parabolic equations (in $n$ dimensions) using a different and more general method based on the maximum principle. We also show that $U(t) \rightarrow 0$ exponentially fast as $t \rightarrow \infty$. Finally, we establish the strict monotonicity of $U(t)$ for all $t>0$; this implies, in particular, that $U(t)$ does not vanish in finite time. For completeness we also prove the existence and uniqueness of $u(x, t)$.

2. $U(t)$ is decreasing. Let

$$
A \equiv \sum_{i, j=1}^{n} a_{i j}(x) \frac{\partial^{2}}{\partial x_{i} \partial x_{j}}+\sum_{i=1}^{n} b_{i}(x) \frac{\partial}{\partial x_{i}}+c(x)
$$

${ }^{*}$ Received August 7, 1984.

${ }^{1}$ This work is partially supported by National Science Foundation grant MCS 79715171. 
be a uniformly elliptic operator in a bounded domain $\Omega$ in $\mathbf{R}^{n}$, that is,

$$
\sum a_{i j}(x) \xi_{i} \xi_{j} \geqslant \mu|\xi|^{2}, \quad x \in \Omega, \xi \in \mathbf{R}^{n},
$$

where $\mu$ is a positive constant, and assume that, for some $0<\alpha<1$,

$$
\begin{gathered}
a_{i j}, b_{i}, c \text { belong to } C^{\alpha}(\bar{\Omega}), \\
c(x) \leqslant 0,
\end{gathered}
$$

for every $x \in \partial \Omega$ there exists a barrier for $A$;

the last condition is satisfied if, for instance, $\partial \Omega$ is in $C^{2}$ or if $\Omega$ is convex.

Let $f(x, y)$ be a continuous function defined for $x \in \partial \Omega, y \in \bar{\Omega}$, and assume that

$$
\theta(x) \equiv \int_{\Omega}|f(x, y)| d y \leqslant \rho<1 \quad \forall x \in \partial \Omega .
$$

For any $0<T \leqslant \infty$ set

$$
\Omega_{T}=\Omega \times\{0<t<T\} .
$$

Consider the parabolic problem:

$$
\begin{gathered}
\frac{\partial u}{\partial t}-A u=0 \quad \text { in } \Omega_{\infty}, \\
u(x, 0)=u_{0}(x) \quad \text { if } x \in \Omega, \\
u(x, y)=\int_{\Omega} f(x, y) u(y, t) d y \quad \text { if } x \in \partial \Omega, 0<t<\infty,
\end{gathered}
$$

where the initial data $u_{0}$ satisfies

$$
u_{0} \not \equiv 0, \quad u_{0} \in C(\bar{\Omega}) .
$$

THEOREM 2.1. There exists a unique solution $u$ of $(2.6)-(2.8)$ such that $u \in C\left(\bar{\Omega}_{\infty}\right)$.

Proof. It suffices to prove existence and uniqueness in $\Omega_{T}$, for any $T<\infty$. Define a sequence $u_{m}(x, t)$ inductively as follows: (i) $u_{0}(x, t) \equiv u_{0}(x)$; (ii) given $u_{m}$, let

$$
\tilde{u}_{m+1}(x, t)=\int_{\Omega} f(x, y) u_{m}(y, t) d y \quad(x \in \partial \Omega)
$$

and let $u_{m+1}$ be the solution of

$$
\begin{gathered}
\left(\frac{\partial}{\partial t}-A\right) u_{m+1}=0 \quad \text { in } \Omega_{T}, \\
u_{m+1}=\tilde{u}_{m+1} \quad \text { on } \partial \Omega \times(0, T), \\
u_{m+1}(x, 0)=u_{0}(x) \quad \text { if } x \in \Omega .
\end{gathered}
$$

Conditions (2.1)-(2.4) ensure that $u_{m+1}$ exists and is continuous in $\bar{\Omega}_{T}$ (provided the same is true of $u_{m}$ ). Further, since

$$
\begin{gathered}
\left(\frac{\partial}{\partial t}-A\right)\left(u_{m+1}-u_{m}\right)=0 \quad \text { in } \Omega_{T} \\
\left(u_{m+1}-u_{m}\right)(x, 0)=0 \quad \text { if } x \in \Omega
\end{gathered}
$$


the maximum principle [5, Chapter 2] gives

$$
\begin{aligned}
\sup _{\Omega_{T}}\left|u_{m+1}(x, t)-u_{m}(x, t)\right| & =\sup _{\substack{x \in \partial \Omega \\
0 \leqslant t \leqslant T}}\left|\int_{\Omega} f(x, y)\left(u_{m}(y, t)-u_{m-1}(y, t)\right) d y\right| \\
& \leqslant \rho \sup _{\Omega_{T}}\left|u_{m}-u_{m-1}\right|,
\end{aligned}
$$

or

$$
\sup _{\Omega_{T}}\left|u_{m+1}-u_{m}\right| \leqslant C \rho^{m} \quad\left(C=\sup _{\Omega_{T}}\left|u_{1}-u_{0}\right|\right) .
$$

It follows that $\left\{u_{m}\right\}$ is a uniform Cauchy sequence and, by standard theory [5], $u \equiv \lim u_{m}$ is the solution of (2.6)-(2.8).

Finally, if $v$ is another solution then [cf. (2.10)]

$$
\sup _{\Omega_{T}}|v-y| \leqslant \rho \sup _{\Omega_{T}}|v-u|
$$

giving $v \equiv u$.

Set

$$
U(t)=\max _{x \in \bar{\Omega}}|u(x, t)|
$$

THEOREM 2.2. The function $U(t)$ is monotone decreasing in $t$.

Proof. If the assertion is not true then there exists a $t_{0}>0$ and sequences of positive numbers $\varepsilon_{m} \downarrow 0, \delta_{m} \downarrow 0$ such that

$$
\delta_{m}+\max _{x \in \bar{\Omega}}\left|u\left(x, t_{0}\right)\right|<\max _{x \in \bar{\Omega}}\left|u\left(x, t_{0}+\varepsilon_{m}\right)\right| .
$$

Without loss of generality we may assume that

$$
\max _{x \in \bar{\Omega}}\left|u\left(x, t_{0}+\varepsilon_{m}\right)\right|=\max _{x \in \bar{\Omega}} u\left(x, t_{0}+\varepsilon_{m}\right) \geqslant-\min _{x \in \bar{\Omega}} u\left(x, t_{0}+\varepsilon_{m}\right) .
$$

Choose $x_{m} \in \Omega$ such that

$$
u\left(x_{m}, t_{0}+\varepsilon_{m}\right)>\max _{x \in \bar{\Omega}} u\left(x, t_{0}+\varepsilon_{m}\right)-\delta_{m} .
$$

Then, by (2.12)

$$
u\left(x_{m}, t_{0}+\varepsilon_{m}\right)>\max _{x \in \bar{\Omega}}\left|u\left(x, t_{0}\right)\right| .
$$

Applying the strong maximum principle to $u$ in $\Omega \times\left(t_{0}, t_{0}+\varepsilon_{m}\right)$ and using (2.14) we conclude that there exist $y_{m} \in \partial \Omega$ and $\tilde{\varepsilon}_{m} \in\left(0, \varepsilon_{m}\right)$ such that

$$
u\left(x_{m}, t_{0}+\varepsilon_{m}\right)<u\left(y_{m}, t_{0}+\tilde{\varepsilon}_{m}\right) \text {. }
$$

But, by (2.8) and (2.5),

$$
\begin{aligned}
u\left(y_{m}, t_{0}+\tilde{\varepsilon}_{m}\right) & \leqslant \int_{\Omega}\left|f\left(y_{m}, x\right)\right|\left[\max _{x \in \bar{\Omega}}\left|u\left(x, t_{0}+\tilde{\varepsilon}_{m}\right)\right|\right] d x \\
& \leqslant \rho\left\{\max _{x \in \bar{\Omega}}\left|u\left(x, t_{0}\right)\right|+\eta_{m}\right\}
\end{aligned}
$$


where $\eta_{m} \rightarrow 0$ by the continuity of $u$. Substituting this into (2.15) and recalling (2.14), we get

$$
u\left(x_{m}, t_{0}+\varepsilon_{m}\right) \leqslant \rho u\left(x_{m}, t_{0}+\varepsilon_{m}\right)+\eta_{m}
$$

or $(1-\rho) u\left(x_{m}, t_{0}+\varepsilon_{m}\right) \leqslant \eta_{m}$. Taking $m \rightarrow \infty$ we deduce, upon using (2.14), that

$$
u\left(x, t_{0}\right) \equiv 0 .
$$

By the uniqueness part of Theorem 2.1 it follows that $u(x, t)=0$ if $t>t_{0}$, a contradiction to (2.12).

THEOREM 2.3. There exist positive constants $C, \gamma$ such that

$$
U(t) \leqslant C e^{-\gamma t} \text { for all } t>0 .
$$

Proof. Let $\left|u_{0}\right| \leqslant M$. The proof of existence shows that $\left|u_{m}\right| \leqslant M$ and therefore also $|u| \leqslant M$ in $\Omega_{\infty}$. Hence, if $y \in \partial \Omega, t>0$,

$$
|u(y, t)| \leqslant \int_{\Omega}|f(y, x) u(x, t)| d x \leqslant \rho M .
$$

By standard results on the asymptotic behavior of the solution of parabolic equations [5, Chapter 6] it follows that

$$
\limsup _{x \in \Omega, t \rightarrow \infty}|u(x, t)| \leqslant \rho M .
$$

Hence, if $\rho_{*}=(\rho+1) / 2$ then

$$
|u(x, t)| \leqslant \rho_{*} M \quad \text { if } x \in \Omega, t>t_{1}
$$

for some large enough $t_{1}$. Similarly, we obtain by induction

$$
|u(x, t)| \leqslant\left(\rho_{*}\right)^{m} M \quad \text { if } t>m t_{1},
$$

so that

$$
U(t) \leqslant\left(\rho_{*}\right)^{m} M \quad \text { if } m t_{1} \leqslant t \leqslant(m+1) t_{1}
$$

It follows that

$$
U(t) \leqslant \frac{M}{\rho_{*}}\left(\rho_{0}\right)^{m+1} \leqslant \frac{M}{\rho_{*}}\left(\rho_{*}\right)^{t / t_{1}}
$$

from which (2.17) follows.

\section{3. $U(t)$ is strictly decreasing.}

Lemma 3.1. There exists a $0<T_{*} \leqslant \infty$ such that $U(t)$ is strictly decreasing if $0<t<T_{*}$ and $U(t) \equiv 0$ if $t>T_{*}$.

Proof. Suppose $U(t)$ is not strictly decreasing for all $t>0$. Then there exist $0<t_{0}<t_{1}$ $<\infty$ such that

$$
U(t)=U\left(t_{0}\right)=U\left(t_{1}\right) \text { for all } t_{0}<t<t_{1} .
$$

In order to prove the lemma it suffices to show that for any such $t_{0}, t_{1}$ we have $U\left(t_{1}\right)=0$. We shall assume that

$$
U\left(t_{1}\right)>0
$$

and derive a contradiction. 
By the maximum principle we then have, for any $x \in \Omega$,

$$
\begin{aligned}
\left|u\left(x, t_{1}\right)\right| & \left.\leqslant \max _{y \in \partial \Omega, t_{1} \leqslant s \leqslant t}|u(y, s)|, \max _{\bar{x} \in \bar{\Omega}}\left|u\left(\bar{x}, t_{0}\right)\right|\right\} \\
& =\max _{t_{0} \leqslant t \leqslant t_{1}} U(t)=U\left(t_{1}\right)=\max _{\bar{x} \in \bar{\Omega}} u\left(\bar{x}, t_{1}\right) \\
& =\max _{y \in \partial \Omega} u\left(y, t_{1}\right)=u\left(y_{1}, t_{1}\right)
\end{aligned}
$$

for some $y_{1} \in \partial \Omega$, where for definiteness we have assumed that

$$
U\left(t_{1}\right)=\max _{\bar{x} \in \bar{\Omega}} u\left(\bar{x}, t_{1}\right) \geqslant-\min _{\bar{x} \in \bar{\Omega}} u\left(\bar{x}, t_{1}\right) .
$$

Hence

$$
\begin{aligned}
u\left(y_{1}, t_{1}\right) & =\int_{\Omega} f\left(y_{1}, x\right) u\left(x, t_{1}\right) d x \leqslant \rho \max _{x \in \bar{\Omega}}\left|u\left(x, t_{1}\right)\right| \\
& \leqslant \rho u\left(y_{1}, t_{1}\right) .
\end{aligned}
$$

Since $\rho<1$ it follows that $u\left(y_{1}, t_{1}\right)=0$ and, by (3.2), $U\left(t_{1}\right)=0$, which contradicts (3.1).

In the sequel we impose the following additional conditions:

$$
\begin{aligned}
& a_{i j} \in C^{1+\alpha}(\bar{\Omega}), \\
& \partial \Omega \text { is in } C^{2+\alpha},
\end{aligned}
$$

and there exists an extension of $f$ into a function $f(x, y)$ defined for all $x \in \bar{\Omega}, y \in \bar{\Omega}$ such that

$$
f(x, y) \in C^{2+\alpha}(\bar{\Omega} \times \bar{\Omega})
$$

We can clearly redefine $f$, if necessary, such that also

$$
\theta(x) \equiv \int_{\Omega}|f(x, y)| d y \leqslant \rho_{0}<1 \quad \text { for all } x \in \bar{\Omega} .
$$

LEMMA 3.2. For any $x \in \Omega$ the function $u(x, t)$ is analytic in $t$, for $0<t<\infty$.

Proof. Introduce the function

$$
V(x, t)=u(x, t)-\int_{\Omega} f(x, y) u(y, t) d y .
$$

We can solve $u$ in terms of $U$, at least formally, by

$$
u(x, t)=V(x, t)+\sum_{m=0}^{\infty} \int_{\Omega} f^{(m)}(x, y) V(y, t) d y
$$

where $f^{(0)}(x, y)=f(x, y)$ and

$$
f^{(m)}(x, y)=\int_{\Omega} f^{(m-1)}(x, z) f(z, y) d z=\int_{\Omega} f(x, z) f^{(m-1)}(z, y) d z .
$$

In view of (3.7)

$$
\left|f^{(m)}(x, y)\right| \leqslant \rho_{0} \sup _{z \in \Omega}\left|f^{(m-1)}(z, y)\right|,
$$

from which we deduce that

$$
\sup _{x, y}\left|f^{(m)}(x, y)\right| \leqslant C\left(\rho_{0}\right)^{m-1}
$$


It follows that the series $\sum f^{(m)}(x, y)$ is uniformly convergent; in fact, it even converges in the $C^{2+\alpha}(\bar{\Omega} \times \bar{\Omega})$ sense. We can thus write

$$
u(x, t)=V(x, t)+\int_{\Omega} F(x, y) V(y, t) d y
$$

with $F$ in $C^{2+\alpha}$.

The function $V$ satisfies:

$$
\begin{aligned}
V_{t}-A V= & -\int_{\Omega} f(x, y) u_{t}(y, t) d y+\int_{\Omega}(A f(x, y)) u(y, t) d y \\
= & -\int_{\Omega} f(x, y)\left[\sum a_{i j}(y) \frac{\partial^{2} u}{\partial y_{i} \partial y_{j}}+\sum b_{i}(y) \frac{\partial u}{\partial y}+c(y) u\right] d y \\
& +\int_{\Omega}\left[\sum a_{i j}(x) \frac{\partial^{2} f}{\partial x_{i} \partial x_{j}}+\sum b_{i}(x) \frac{\partial f}{\partial x_{i}}+c(x) f\right] u(y, t) d y .
\end{aligned}
$$

Writing

$$
\begin{aligned}
-\int_{\Omega} f(x, y) a_{i j}(y) \frac{\partial^{2} u(y)}{\partial y_{i} \partial y_{j}} d y=-\int_{\partial \Omega} f(x, y) & a_{i j}(y) \nu_{i} \frac{\partial u(y, t)}{\partial y_{j}} d S_{y} \\
& +\int_{\Omega} \frac{\partial}{\partial y_{i}}\left(f(x, y) a_{i j}(y)\right) \frac{\partial u(y, t)}{\partial y_{j}} d y
\end{aligned}
$$

we see that

$$
V_{t}-A V=G \text { in } \Omega_{\infty},
$$

where

$$
\begin{aligned}
G=\sum \int_{\partial \Omega} \tilde{\alpha}_{j}(x, y) \frac{\partial u(y, t)}{\partial y_{j}} d S_{y} & +\sum \int_{\Omega} \tilde{\beta}_{j}(x, y) \frac{\partial u(y, t)}{\partial y_{j}} d y \\
& +\int_{\Omega} \tilde{\gamma}(x, y) u(y, t) d y
\end{aligned}
$$

and $\tilde{\alpha}_{j}, \tilde{\beta}_{j}, \tilde{\gamma}$ are in $C^{\alpha}$. Substituting $u$ and $\partial u / \partial y_{j}$ from (3.12) we find that

$$
\begin{aligned}
G=G(V)=\sum \int_{\partial \Omega} \alpha_{j}(x, y) \frac{\partial V(y, t)}{\partial y_{j}} d S_{y}+\sum \int_{\Omega} \beta_{j}(x, y) \frac{\partial V(y, t)}{\partial y_{j}} d y \\
+\int_{\Omega} \gamma(x, y) V(y, t) d y,
\end{aligned}
$$

where $\alpha_{j}, \beta_{j}, \gamma$ are in $C^{\alpha}$.

Note that

$$
V(x, t)=0 \quad \text { if } x \in \partial \Omega, t>0,
$$

and

$$
V(x, 0)=V_{0}(x) \text { if } x \in \Omega, V_{0} \in C(\bar{\Omega}) .
$$


The parabolic problem (3.13), (3.15), (3.16) is not a standard one since $G$ is a nonlocal (linear) operator of $V$. Nevertheless, one can still apply Schauder-type estimates in order to derive inductively that, for any $0<T<\infty, 1 \leqslant m<\infty$,

$$
\left|D_{t}^{m} V(y, t)\right| \leqslant \frac{K_{0} K^{m-1}}{t^{m-1+n / 2}} \frac{m !}{m^{2}} \quad \text { in } \Omega_{T}
$$

with similar bounds on $D_{t}^{m-1} D_{y}^{2} V(y, t)$ and on the corresponding Hölder coefficients. Indeed, in [6] we considered the parabolic problem

$$
\begin{gathered}
v_{y y}-v_{t}=v_{y}(1, t) v_{y} \quad \text { in }\{0<y<1,0<t<T\}, \\
v(0, t)=v(1, t)=0 \quad \text { if } 0<t<T,
\end{gathered}
$$

and established estimates of the type (3.17). The proof can be extended to parabolic equations with variable coefficients in $n$-dimensional domains [and with $v_{y}(1, t) v_{y}$ replaced by $G(V)$ ]. In fact, the basic estimates from which one deduces (3.17) (for $v$ ) are the interior-boundary Schauder estimates stated in Theorem 1.1 of [6]. This theorem can be extended to general parabolic operators in general domains by a standard argument used to derive the Schauder estimates for variable coefficients and general domains, once they have been proven for constant coefficients in rectangular domains; see [5, Chapter 4]. Since the calculations are routine, we omit the details.

The assertion (3.17) now follows from the interior-boundary Schauder estimates in a way similar to that for $v$ in [6].

From (3.17) and (3.12) we see that $u(x, t)$ is analytic in $t$, for each $x \in \Omega$.

THEOREM 3.3. If (3.4)-(3.6) hold, then $U(t)$ is strictly decreasing in $t$ for all $t>0$.

Proof. If the assertion is not true then, by Lemma 3.1, $u(x, t) \equiv 0$ for all $t>T_{*}$. By Lemma 3.2 it then follows that $u(x, t) \equiv 0$ in $\Omega_{\infty}$, a contradiction to $u_{0} \equiv \equiv 0$.

REMARK. Theorems 2.1-2.3 and Lemma 3.1 extend to the case where the coefficients of $A$ also depend on $t$. Lemma 3.2 and Theorem 3.3 also extend to this case provided the coefficients are analytic in $t$.

\section{REFERENCES}

[1] B. A. Boley and J. H. Weiner, Theory of thermal stresses, Wiley, New York, 1960

[2] D. E. Carlson, Linear thermoelasticity, in Encyclopedia of physics, VI a/2, Springer, Berlin, 1972

[3] W. A. Day, Extensions of a property of the heat equation to linear thermoelasticity and other theories, Quart. Appl. Math. 40, 319-330 (1982)

[4] W. A. Day, A decreasing property of solutions of parabolic equations with applications to thermoelasticity, Quart. Appl. Math. 41, 468-475 (1983)

[5] A. Friedman, Partial differential equations of parabolic type, Prentice-Hall, Englewood Cliffs, N. J. (reprinted by Krieger, Malabar, Fla., 1983)

[6] A. Friedman, Analyticity of the free boundary for the Stefan problem, Arch. Rat. Mech. Anal. 61, 97-125 (1976) 